ACTA SCIENTIFIC MICROBIOLOGY (ISSN: 2581-3226)

Volume 4 Issue 10 October 2021

Review Article

\title{
Role of Colostrum in Immune-Protection and Disease Prevention in Neonates and their Post Natal Life
}

\section{Ravi Kant Upadhyay*}

Department of Zoology, Deen Dayal Upadhyaya Gorakhpur University, Gorakhpur, $U P$, India

*Corresponding Author: Ravi Kant Upadhyay, Department of Zoology, Deen Dayal Upadhyaya Gorakhpur University, Gorakhpur, UP, India.
Received: August 16, 2021

Published: September 08, 2021

(C) All rights are reserved by Ravi Kant

Upadhyay.

\begin{abstract}
Colostrum is naturally secreted nutritionally rich thick biological fluid that contains various bioactive molecules such as proteins, carbohydrates, fats, hormones, growth factors, vitamins, lectoferrins, and good repertoire of immunoglobulins which play important role in innate immune defense in new born infants. It contains disease protective and growth promoting micro and macronutrients. Colostrum or milk suckling by mammalian new born babies is a unique phenomenon as it passively transfers important growth factors, which are highly essential for maturation and post natal development. Presence of IgA, IgG and IgM in colostrums protects new born babies from gut mucosal microbial infection. It is highly beneficial for health of sport persons and has high therapeutic value. Commercial storage of colostrums can be used for long term treatment of preterm babies, immunocompromised patients and elderly. Hence, sterilize colostrum collection systems be developed for its clinical and therapeutic use. More specially, for preterm babies constitutive colostrum feeds can be generated with infant formula to protect them from microbial gut infection mainly colonizing bacteria at larger scale. Present review is emphasizing benefits of breast feeding in newborn infants and sketches out immune-protective, nutritional and growth promoting properties of colostrums.
\end{abstract}

Keywords: Colostrum; Nutritional Value; Immunoglobulins; Innate Immune Defense; Disease Prevention and Therapeutic Uses

\section{Introduction}

Colostrum is secreted from mammary glands of lactating mammals just after birth of neonates or babies/infants. It is first secreted by post partum mammals and differs in composition from milk. It is a good source of micro and macronutrients, growth factors and immunoglobulins. Colostrums is a good source of various antibodies mainly IgG that has great therapeutic value [1]. Feeding of colostrums support systemic immune function and maintain gut function in newborn babies [2]. Human beings also get colostrums from external source mainly from cows, goat, buffalo, camel, sheep, and yak and other livestock. Suckling of first milk or colostrums passively transfers innate immune functions. In pregnant females antibodies circulate in blood stream which cross placenta and provide protection against pathogens inside uterus of mother. All neonates live inside sterilize environment of uterus inside several layers of membranes and their protection is ensured due to various circulating immunoglobulins, but soon after birth, new born come in adverse environment or in infectious surroundings all of a sudden. It is amazing and nature's boon that colostrums feeding/ breast feeding provide safety shield to post natal life. It possesses important components such as antibodies and antimicrobial peptides of innate or germ line immune defense [3]. Colostrum suckled by newborns or young ones after birth imparts wider defense against pathogens [4]. It accelerates growth because of higher utilization of substrates and ample energy supply in neonates [5]. Therefore, breast feeding up to six month after birth is highly im- 
portant and essential for infant health and survival [6]. Both colostrums and milk are the best source for newborn nutrition until at least six months.

Consumption of colostrums based dairy items provides protection against infectious agents. Its use is good for health of infants, children, elderly, athletes and immune compromised patients [7]. Both human and bovine colostrums are highly used in gastrointestinal infections and lower down neoplastic activity [8]. It is not only good for newborn babies but it improves mother's health; it reduces overweight and obesity in mothers. Colostrum shows laxative activity and assist in first stool flow in new born, it also lower down bile salts specially bilirubin formed from dead RBCs and prevent jaundice. It also contains lactoferrins and hemopexin both bind heme iron and show anti-oxidant activity [9,10]. Colostrum secreted from mothers is easily absorbed by the lymphoid tissues of the oropharynx [11]. Colostrum fats are less saturated and posses more appropriate LQIs values [12].

After birth of preterm babies lack of maternal milk secretion or a limited supply is seen in mothers. In such condition infant formula much similar to the first enteral feed is provided through artificial methods. But it is responsible for necrotizing enterocolitis (NEC), and infection [13]. The oropharyngeal administration of colostrum from external sources is highly beneficial for premature newborns [11]. It instantly provides nutrients, energy, vitamins, various growth factors and immunoglobulins. It is energy rich natural growth promoting functional food. Hence, breast feeding is good for health of new born babies because it essentially transfer passive immunity that protects against infection [14].

\section{Composition}

Colostrum is yellow colored thick liquid having salty taste and aroma of buttermilk. It contains good nutritional supplement that support growth and survival of infants and children. Colostrum is nutritionally rich and contains micro and macro-nutrients mainly glycans, minerals and vitamins, proteins, carbohydrates and fats which support infant growth and development [15]. It contains various immunoglobulins i.e. IgG (25 gm/100 mL), IgA (1.5 - 3.5 $\mathrm{gm} / 100 \mathrm{gm})$ and IgM (0.5 - $1.0 \mathrm{gm} / 100 \mathrm{gm})$. It also contains important growth factors IGF-1 and 2, EGF, FGF, PDGF, EGF1, VEGF, TGF, etc. IgA is the predominant immunoglobulin in human milk but its level found lower than colostrum. In human lactating mothers transfer of IgA antibodies from the intestine to the mammary gland occurs through enteromammaric link. Mother's milk contains important defense making factors such as lactoferrin, lysozyme, and macrophages and granulocytes operate phagocytosis. Secretory IgA is an important part of the host defense at the mucosal level in the gastrointestinal, as well as the respiratory and genito-urinary tracts. Breast-feeding baby gets 0.25 - 0.5 grams per day secretory IgA antibodies via the passive transfer through suckling of milk. It is highly useful for babies born with low levels of IgA but its level slowly rises according to milk suckling duration. It is only possible through long term or atleast six months of baby breastfeeding.

Colostrum contains lactose (20 gm/100 gm) and glycoproteins lactoferrin and transferrin approximately $0.1 / 100 \mathrm{gm}$ in concentration (Table 1 and figure 1). These iron binding glycoproteins show antimicrobial activity and maintain innate immune defense in infants assist in operation against pathogens. These make attack on pathogens much better than neutrophils. Colostrum contains melatonin which reduces maternal weight and boost up neonatal defense [16]. Colostrum contains immunoglobulins, lysozyme, alpha lactalbumin, beta globulins, fats, vitamins and polyunsaturated fatty acids and proline rich polypeptides [17] (Figure 1). Cow milk has all different factors much similar to mothers milk (Table 2).

\begin{tabular}{|c|c|c|}
\hline Components & Colostrum in $\mathbf{( g / 1 0 0 g )}$ & Milk (g/100g) \\
\hline Proteins & $60-70$ & 3.2 \\
\hline Carbohydrates & $15-20$ & 6.0 \\
\hline Total fats & $<0.2$ & 3.2 \\
\hline Immunoglobulins & & \\
IgG & 25 & $10-15$ \\
IgA & $1.5-3.5$ & $0.7-1.0$ \\
IgM & $0.5-1.0$ & $0.25-0.50$ \\
\hline Lactoferrin & Approximately 0.1 & $0.3-4.2$ \\
\hline Lactose & $15-20$ & 4.9 \\
\hline Vitamin A $\mu$ g/dl & 294.0 & 34.0 \\
\hline Minerals\% & 1.11 & 0.74 \\
\hline Total solids & 23.9 & 12.5 \\
\hline
\end{tabular}

Table 1: Composition of colostrums and human milk. 


\begin{tabular}{|c|c|c|}
\hline Main constituent & Range (\%) & Mean (\%) \\
\hline Water & $85.5-89.5$ & 87.0 \\
\hline Total solids & $10.5-14.5$ & 13.0 \\
\hline Fat & $2.5-6.0$ & 4.0 \\
\hline Proteins & $2.9-5.0$ & 3.4 \\
\hline Lactose & $3.6-5.5$ & 4.8 \\
\hline Minerals & $0.6-0.9$ & 0.8 \\
\hline
\end{tabular}

Table 2: Composition of cow milk.

*Cow milk has much similar composition to mothers milk.

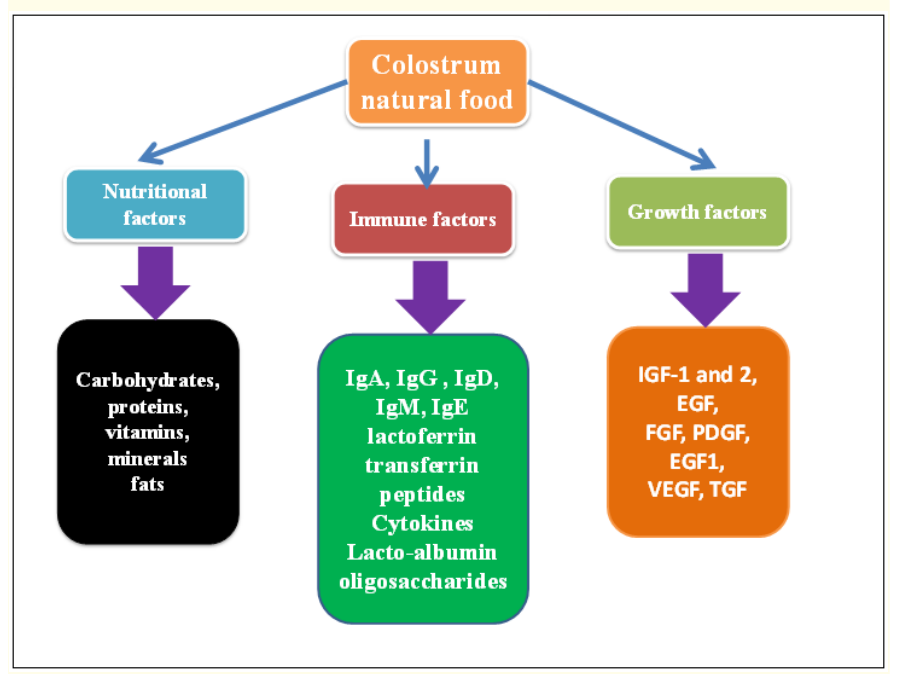

Figure 1: Showing nutritional, immune factors and growth promoting factors found in colostrum.

Colostrum contains cytokines, complements, $\beta$ TGF and immunoglobulin (IgA) which assist in disease prevention. Bovine colostrum also contains cytokines which increase B and T cell maturation and accelerate endogenous antibody production (Figure 1). They also play a major role in regulation of epithelial cell growth, development and proliferation [18]. These essentially induce immunity and assist in fighting microbial infection in throat, gut and lungs in newborns $[18,19]$. Several factors affect colostrum quality and yield. In humans and bovine colostrum consumption increases the immune protection in vaccinated cases [20]. Colostral immunoglobulin levels differ according to hormonal level of mothers [21]. It could be maximized by management practices [22].
Disease prevention

Bovine colostrum (BC) is a biological fluid rich in various bioactive molecules which help in disease prevention mainly exert anti-inflammatory and anti-infective functions [18]. Colostrum is highly beneficial in many gastrointestinal disorders and diseases [23]. It protects from bacterial infection in mucosal sites due to release of IgA antibodies [24]. It also lowers down gastrointestinal carriage of uropathogenic Escherichia coli [25] and repairs intestinal injury [26]. This is one of the important reason that breast fed infants show lower risk of gastrointestinal tract infection [8]. Supplementation of colostrums with oligosaccharides on serum IgG concentration increases the absorption efficiency [27]. Colostrum is also found beneficial in enzootic bovine leukosis (EBL) caused by Bovine leukemia virus (BLV). More often, colostral antibodies neutralize against BLV antigens, and conferred as anti-BLV gp51 antibody [28]. Colostrum pretreatment reduces the intestinal damages and clinical signs of the TNBS (2,4,6 trinitrobenzene sulfonic acid) induced colitis [23].

Colostrum also shows preventive effects against human rotavirus [29]. Its oral dose significantly enhances the release of immunoglobin IgG [30]. Colostrum fed neonatal calves posses passively transferred antibodies which were found active against bovine respiratory syncytial virus [31]. Colostrum with low concentration of immunoglobulins did not show sizable immunity in calves [32]. Colostrum treatment increase level of HIV-1 specific IGg1 secreting memory B cells [33] which can make anti-viral defense against HIV-1 infected mothers [34] (Figure 2). Porcine milk with colostrums contains determinants of early immune programming that provide protection from $C$ difficule infection [35]. Colostrumacquired maternal antibodies make systemic and mucosal immune responses to rotavirus [31] and Zika virus (ZIKV) and Usutu virus (USUV) [36]. From researches it is proved that human milk (HM) induce formation of extracellular vesicles (EVs) and glycosaminoglycans (GAGs) which show protective effect against viruses [36] (Figure 2).

\section{Therapeutic effects}

In all mammals milk is essential for the initial development of new born babies, it provides all required nutritional supplements and is a great source of commensal bacteria. Colostrum secreted in human and bovine shows many therapeutic applications. Both colostrum and mature milk assist in colonization of gut microbiota 


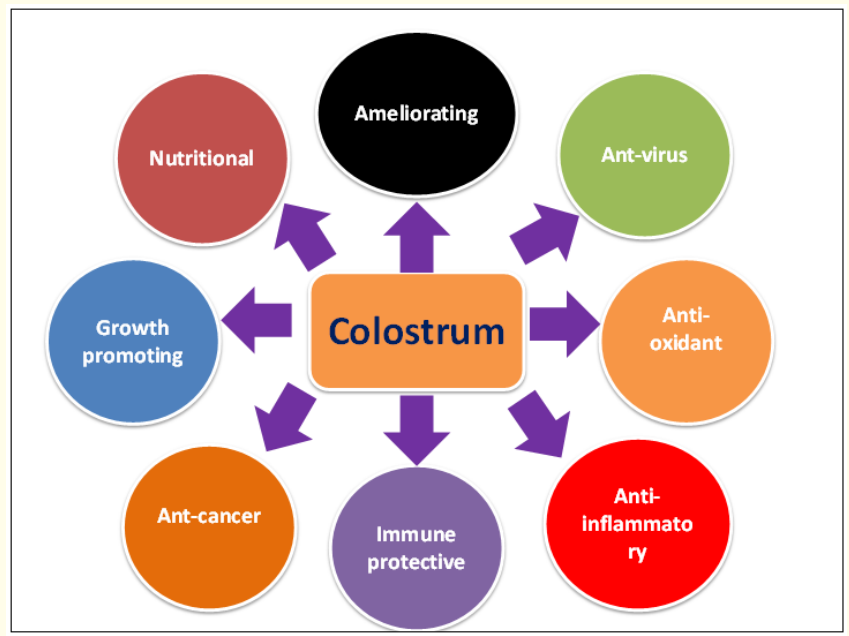

Figure 2: Showing nutritional, growth promoting and therapeutic effects of colostrum.

extend potential health promotion effects in neonates [37]. It found highly effective in prevention of chronic intestinal inflammation [38]. It could be used for lower down the risk of human gastrointestinal diseases [8] and boost up human health [39]. Colostrum made products increase systemic IGF 1 level [40]. It is found highly effective in necrotizing enterocolitis (NEC), and infection [13]. Colostrum shows ameliorative effects against DMBA hepatotoxicity in albino rats [41]. Bovine Colostrum also lowers down polycyclic aromatic hydrocarbons-induced hepatotoxicity [41] (Figure 2).

\section{Innate immune functions}

Human colostrum (HC) is a rich source of immune mediators that play role in immune defences of a newly born infant. The mediators include transforming growth factor $\beta$ (TGF- $\beta$ ) which exists in three isoforms that regulate cellular homeostasis and inflammation [42]. These induce or suppress immune responses, limit $\mathrm{T}$ helper 1 cell (Th1) reactions and stimulate secretory immunoglobulin $\mathrm{A}$ (IgA) production. Human milk TGF- $\beta$ also decrease apoptosis of intestinal cells and suppress macrophage cytokine expression [42]. Colostral antibodies against BLV infection would be conferred by anti-BLV gp51 antibody. The high antibody titer of colostral whey suggests that colostral whey could be a potential source of antibodies with a low risk of infection in neonatal calves [28]. Colostrum supplements in piglets significantly increase $\mathrm{T}$ - lymphocyte cells mainly effective memory CD4+ T cells, increase immunoglobulin levels and causes neutralization of infectious agents [43]. Colostrum of young mothers contains ample concentration of interleukin (IL)-1 $\beta$ and IL-6 compared to adolescent mothers [44]. Bokashi is fed to dairy cattle that increase the level of pro-inflammatory cytokines, TNF and IL 6. These cytokines assist stimulating cell mediated immune defense. Bokashi also exerts immune regulatory effects on the two cells and significantly increase the expression of regulatory $\mathrm{T}$ cells and protect the fetus from congenital infection [45] (Figure 2).

\section{Energy booster}

Freezed and stored colostrums can be used for preparation of high energy foods for growing infants, children, adults and sports persons. Use of colostrums or colostrums based food items provide strength, relieve stress, muscular and cytoskeletal stiffness [46]. Colostrum constituents accelerate protein synthesis, cellular proliferation and tissue repair. For long term dietary use colostrums can be stored, its supplements favor disease prevention and enhance immune protection in newborns [47]. Use of bovine colostrum (BC) as therapeutic agent immune system dysfunctions often associated with alternations in the microbiota, which contribute to the development of chronic intestinal inflammation [38]. Commercially bovine colostrums is available in market contains growth hormones and digestive enzymes. Bovine colostrum use is highly beneficial to sport persons mainly athletes [48]. It increases the physical performance it boosts up immunity, increase physical fitness and restore body metabolism [40]. The colostrum collection and its freezing are essential as it is good for clinicians and patients [49]. There is a need to aware dairy producers for quality enhancement of colostrum and its proper storage and management [50].

\section{Conclusion}

Colostrum is nutritionally rich natural product secreted from breasts of females, it is a good supplement that promotes infant growth and has several health benefits. Colostrum is a good source of potentially bioactive molecules which support neonatal development and systemic lactogenic immune transfer of immunoglobulins that assists in maintaining of innate immune defense. It contains immunoglobulins which protect the new born from infection of gut, and lungs. It contains many bioactive constituents such as 
growth factors, and oligosaccharides which enhance nutrient absorption, and work as prebiotics and assist in colonization of useful bacteria in the gut of infants, children and adults. No doubt breast feeding is good for overall growth and health of infants. It increases disease prevention, boost up immunity, survival and provide vitality to the infants. Babies devoid of colostrum or lack of colostrums remain weak and under growth. Use of colostrums improves respiratory and digestive health of infants.

\section{Acknowledgements}

Author is thankful to H.O.D., Department of Zoology for research facilities.

\section{Conflict of Interest}

The authors declare no competing financial interests.

\section{Bibliography}

1. Williams DR., et al. "Effect of Three Colostrum Diets on Passive Transfer of Immunity and Preweaning Health in Calves on a California Dairy following Colostrum Management Training". Veterinary Medicine International 2014 (2014).

2. Li Y., et al. "Bovine Colostrum Before or After Formula Feeding Improves Systemic Immune Protection and Gut Function in Newborn Preterm Pigs". Frontiers in Immunology 10 (2020): 3062.

3. Hurley WL., et al. "Perspectives on Immunoglobulins in Colostrum and Milk". Nutrients 3.4 (2011): 442-474.

4. Sears KT., et al. "Bioactive Immune Components of Anti-Diarrheagenic Enterotoxigenic Escherichia coli Hyperimmune Bovine Colostrum Products". Clinical and Vaccine Immunology 24.8 (2017).

5. Liermann W., et al. "Effects of colostrum instead of formula feeding for the first 2 days postnatum on whole-body energy metabolism and its endocrine control in neonatal calves". Journal of Dairy Science 103.4 (2020): 3577-3598.

6. Sandra M Godden., et al. "Colostrum management for dairy calves". Veterinary Clinics of North America: Food Animal Practice 35.3 (2019): 535-556.

7. Ulfman LH., et al. "Effects of Bovine Immunoglobulins on Immune Function, Allergy, and Infection". Frontiers in Nutrition 5 (2018): 52.
8. Bagwe-Parab S., et al. "Therapeutic Applications of Human and Bovine Colostrum in the Treatment of Gastrointestinal Diseases and Distinctive Cancer Types: The Current Evidence". Frontiers in Pharmacology 11 (2020): 01100.

9. Wakabayashi Hiroyuki., et al. "Inhibition of Iron/Ascorbate-Induced Lipid Peroxidation by an N-Terminal Peptide of Bovine Lactoferrin and Its Acylated Derivatives". Bioscience, Biotechnology, and Biochemistry 63.5 (1999): 955-957.

10. Gutteridge J M and Smith. "Antioxidant protection by haemopexin of haem-stimulated lipid peroxidation". Biochemical Journal 256.3 (1988): 861-865.

11. da Cruz Martins C., et al. "Colostrum oropharyngeal immunotherapy for very low birth weight preterm infants: protocol of an intervention study". BMC Pediatrics 20 (2020).

12. Sinanoglou VJ. "Factors affecting human colostrum fatty acid profile: A case study". PLoS One 12.4 (2017).

13. Li Y., et al. "Bovine Colostrum Before or After Formula Feeding Improves Systemic Immune Protection and Gut Function in Newborn Preterm Pigs". Frontiers in Immunology 10 (2020): 3062 .

14. Jawhara S., et al. "Can Drinking Microfiltered Raw Immune Milk From Cows Immunized Against SARS-CoV-2 Provide Short-Term Protection Against COVID-19?". Frontiers in Immunology 11 (2020).

15. Arslan A., et al. "Bovine Colostrum and Its Potential for Human Health and Nutrition". Frontiers in Nutrition 8 (2020).

16. Tassiane C Morais., et al. "Melatonin Action on the Activity of Phagocytes from the Colostrum of Obese Women". Medicina (Kaunas) 55.10 (2019): 625.

17. Puppel K., et al. "Composition and Factors Affecting Quality of Bovine Colostrum: A Review". Animals (Basel) 9.12 (2019): 1070.

18. Bardanzellu F., et al. "Omics in Human Colostrum and Mature Milk: Looking to Old Data with New Eyes". Nutrients 9.8 (2017): 843.

19. Munblit D., et al. "Levels of Growth Factors and IgA in the Colostrum of Women from Burundi and Italy". Nutrients 10.9 (2018): 1216. 
20. Kasonta R., et al. "Colostrum from Cows Immunized with a Vaccine Associated with Bovine Neonatal Pancytopenia Contains Allo-Antibodies that Cross-React with Human MHC-I, Molecules". PLoS One 9.10 (2014).

21. Kaplan DS., et al. "Colostrum immunoglobulins and oxidative capacity may be affected by infant sex and maternal age and parity". Turkish Journal of Medical Sciences 49.1 (2019): 87-92.

22. Soufleri A., et al. "Evaluation of Factors Affecting Colostrum Quality and Quantity in Holstein Dairy Cattle". Animals (Basel) 11.7 (2021): 2005.

23. Filipescu IE., et al. "Preventive effects of bovine colostrum supplementation in TNBS-induced colitis in mice". PLoS One 13.8 (2018).

24. Sánchez-Salguero E., et al. "Infectious episodes during pregnancy, at particular mucosal sites, increase specific IgA1 or IgA2 subtype levels in human colostrum". Maternal Health, Neonatology and Perinatology 11.5 (2019).

25. Larcombe S., et al. "Hyperimmune bovine colostrum reduces gastrointestinal carriage of uropathogenic Escherichia coli". Human Vaccines and Immunotherapeutics 15.2 (2018): 508513.

26. March DS., et al. "The effect of bovine colostrum supplementation on intestinal injury and circulating intestinal bacterial DNA following exercise in the heat". European Journal of Nutrition 58.4 (2019): 1441-1451.

27. Short DM., et al. "A Randomized Clinical Trial Evaluating the Effects of Oligosaccharides on Transfer of Passive Immunity in Neonatal Dairy Calves". Journal of Veterinary Internal Medicine 30.4 (2016): 1381-1389.

28. Konishi M., et al. "The effectiveness of colostral antibodies for preventing bovine leukemia virus (BLV) infection in vitro". BMC Veterinary Research 14 (2018).

29. Parreño V., et al. "Modulation by colostrum-acquired maternal antibodies of systemic and mucosal antibody responses to rotavirus in calves experimentally challenged with bovine Rotavirus". Veterinary Immunology and Immunopathology 100.1 (2004): 7-24.

30. Civra A., et al. "Colostrum from cows immunized with a veterinary vaccine against bovine rotavirus displays enhanced in vitro anti-human rotavirus activity". Journal of Dairy Science 102.6 (2019): 4857-4869.
31. Ellis JA., et al. "Bovine respiratory syncytial virus-specific IgG1 in nasal secretions of colostrum-fed neonatal calves". Canadian Veterinary Journal 59.5 (2018): 505-508.

32. Drikic M., et al. "Determining the IgG concentrations in bovine colostrum and calf sera with a novel enzymatic assay". Journal of Animal Science and Biotechnology 9 (2018): 69.

33. Sacha., et al. "Restricted isotype, distinct variable gene usage, and high rate of gp120-specificity of HIV-1 Envelope-specific B cells in colostrum compared to those in blood of HIV-1-infected, lactating African women". Mucosal Immunology 8.2 (2015): 316-326.

34. Jeffries TL., et al. "The function and affinity maturation of HIV1 gp120-specific monoclonal antibodies derived from colostral B cells". Mucosal Immunology 9.2 (2016): 414-427.

35. Grześkowiak Ł., et al. "Porcine Colostrum Protects the IPEC-J2 Cells and Piglet Colon Epithelium against Clostridioides (syn. Clostridium) difficile Toxin-Induced Effects". Microorganisms 8.1 (2020): 142.

36. Francese R., et al. "Anti-Zika virus and anti-Usutu virus activity of human milk and its components". PLOS Neglected Tropical Diseases 14.10 (2020).

37. Niyazbekova Z., et al. "Compositional and Functional Comparisons of the Microbiota in the Colostrum and Mature Milk of Dairy Goats". Animals (Basel) 10.11 (2020): 1955.

38. Sienkiewicz M., et al. "Supplementation of Bovine Colostrum in Inflammatory Bowel Disease: Benefits and Contraindications". Advances in Nutrition 12.2 (2020): 533-545.

39. Chandwe K., et al. "Colostrum Therapy for Human Gastrointestinal Health and Disease". Nutrients 13.6 (2021).

40. Glen Davison. "The Use of Bovine Colostrum in Sport and Exercise". Nutrients 13.6 (2021): 1789.

41. Abdelmeguid NE. "Ameliorative effects of colostrum against DMBA hepatotoxicity in rats". Saudi Journal of Biological Sciences 28.4 (2021): 2254-2266.

42. Kociszewska-Najman B., et al. "Does Caesarean Section or Preterm Delivery Influence TGF- $\beta 2$ Concentrations in Human Colostrum?" Nutrients 12.4 (2020): 1095.

43. Forner R., et al. "Distribution difference of colostrum-derived $\mathrm{B}$ and T cells subsets in gilts and sows". PLoS One 16.5 (2021). 
44. Ferrari DVde J., et al. "Profile of pro-inflammatory cytokines in colostrum of nursing mothers at the extremes of reproductive age". PLoS One 15.6 (2020).

45. Laskowska E., et al. "Effect of Multi-Microbial Probiotic Formulation Bokashi on Pro- and Anti-Inflammatory Cytokines Profile in the Serum, Colostrum and Milk of Sows, and in a Culture of Polymorphonuclear Cells Isolated from Colostrum". Probiotics Antimicrobe Proteins 11.1 (2019): 220-232.

46. Playford RJ., et al. "Bovine Colostrum: Its Constituents and Uses”. Nutrients 13.1 (2021): 265.

47. Lisa Robbers., et al. "A Scoping Review of On-Farm Colostrum Management Practices for Optimal Transfer of Immunity in Dairy Calves". Frontiers in Veterinary Science 8 (2021): 668639.

48. Skarpańska-Stejnborn A., et al. "Effects of Long-Term Supplementation of Bovine Colostrum on the Immune System in Young Female Basketball Players. Randomized Trial". Nutrients 13.1 (2020): 118.

49. Alexandria I Kristensen-Cabrera., et al. "A prospective clinical study of Primo-Lacto: A closed system for colostrum collection". PLoS One 13.11 (2018): e0206854.

50. Johnsen JF., et al. "A cross-sectional study of suckling calves' passive immunity and associations with management routines to ensure colostrum intake on organic dairy farms". Acta Veterinaria Scandinavica 61 (2019): 7.

\section{Volume 4 Issue 10 October 2021}

(C) All rights are reserved by Ravi Kant Upadhyay. 